\title{
Measurements of Pulsed 532 nm Laser Breakdown Spectroscopy of Synthesized Magnetite Nanoferrofluid
}

\author{
Mohammad E. Khosroshahii ${ }^{1,2,3}{ }^{*}$, Maryam Tajabadi ${ }^{1,4}$ \\ ${ }^{1}$ Laser and Nanobiophotonics Laboratory, Biomaterial Group, Faculty of Biomedical Engineering, \\ Amirkabir University of Technology, Tehran, Iran \\ ${ }^{2}$ MIS-Electronics, Nanobiophotonics and Biomedical Research Laboratory, Richmond Hill, Canada \\ ${ }^{3}$ Department of Mechanical and Industrial Engineering, University of Toronto, Toronto, Canada \\ ${ }^{4}$ School of Metallurgy and Materials Engineering, Iran University of Science and Technology, Tehran, Iran \\ Email: *khosrom@mie.utoronto.ca
}

How to cite this paper: Khosroshahi, M.E. and Tajabadi, M. (2018) Measurements of Pulsed 532 nm Laser Breakdown Spectroscopy of Synthesized Magnetite Nanoferrofluid. World Journal of Nano Science and Engineering, 8, 39-55.

https://doi.org/10.4236/wjnse.2018.83003

Received: August 3, 2018

Accepted: September 25, 2018

Published: September 28, 2018

Copyright $\odot 2018$ by authors and Scientific Research Publishing Inc. This work is licensed under the Creative Commons Attribution International License (CC BY 4.0).

http://creativecommons.org/licenses/by/4.0/

Open Access

\begin{abstract}
We describe the results of $532 \mathrm{~nm}$ pulse laser-induced breakdown spectroscopy (LIBS) of two samples of magnetite nanoparticles (SPIONs) nanoferrofluid synthesized at room (S1) and elevated temperatures (S2) and at three different laser energy levels and pulse frequency. The size of magnetite nanoparticles, size distribution, magnetic crystalline phase and magnetization were analyzed and measured using transmission electron microscopy (TEM), X-ray diffraction spectroscopy (XRD) and vibrating sample magnetometry (VSM). The SPIONs showed a distribution between $4-22 \mathrm{~nm}$ with a peak about $12 \mathrm{~nm}$ and saturation magnetization of about $65 \mathrm{emu} / \mathrm{g}$. The Saha-Boltzmann analysis of spectra for medium energy level $(1050 \mathrm{~mJ})$ yields plasma temperatures of $(3881 \pm 200) \mathrm{K}$ and $(26,047 \pm 200) \mathrm{K}$ for Fe I and OV as the lowest and highest temperatures respectively. A range of corresponding electron density $\left(\mathrm{Ne}^{-}\right)$of $(0.47-6.80) \times 10^{20},(0.58-8.30) \times 10^{20}$ and $(0.69-9.96) \times 10^{20} \mathrm{~cm}^{-3}$ were determined at 860,1050 and $1260 \mathrm{~mJ}$ respectively using the estimated CCD pictures. The results confirmed a higher elements ratio for S1 than S2 and the signal intensity indicated a non-linear behaviour as a function of pulse frequency with the maximum ratio value at $3 \mathrm{~Hz}$. At higher frequency of $6 \mathrm{~Hz}$ no such turning point was observed. The highest and lowest temperatures corresponded to Fe I and OV respectively. The LIBS technique can be utilized to study, characterize and determine the elements ratio required in most applications involving the synthesizing process.
\end{abstract}

\section{Keywords}

Laser-Induced Breakdown Spectroscopy, Second Harmonic Generation, SPION, 
Nanoferrofluid, Elements Ratio

\section{Introduction}

In recent year much interest has been shown for remote, versatile, fast as well as accurate elemental analysis. Analysis techniques such as laser-ablation inductively coupled mass spectrometry (LA-ICP-MS) [1], X-ray fluorescence spectrometry (XRFS) [2], and secondary ion mass spectrometry (SIMS) [3] are able to resolve smaller element concentration. However, they often require specific sample preparation and under-lab condition operation and have smaller analyzed material capacity per time. Laser-induced breakdown spectroscopy (LIBS), which is considered as a type of atomic emission spectroscopy is based on focusing a short laser pulse on the surface of the sample where the material is ablated via thermal or non-thermal mechanisms. A high density plasma is generated when the laser intensity reaches the breakdown threshold of $\approx 10^{10} \mathrm{Wcm}^{-2}$. The optical emission is then collected and spectroscopically analyzed. It is the different energy levels of each atom that induces different and well quantized energies with narrowband emissions governed by Heisenberg uncertainty principle. When a material is ablated by a short laser pulse, the plume partly contains free electrons, excited atoms and ions, which are produced by strong ionization under an intense external electric field. The spatial free electron density i.e., plasma is produced by multiphoton absorption process where the electrons absorbing the energy of laser beam photons via inverse bremsstrahlung will result in a process known as avalanche or cascade of ionization. When the laser pulse terminates, a few microseconds later the plasma begin to slow down due to collisions with ambient gas and hence cool down during the expansion where the electrons of the atoms and ions at the excited electronic states decay to ground states, hence causing the plasma to emit light with discrete spectral peaks. Both the continuum and discrete line emission indicate the radiative relaxation of different energy levels of the ions and the neutral atoms and that the excited species are mainly generated by ion-electron recombination. The main features of discrete lines characterizing the material are: wavelength, intensity and the shape which depend on structure of the emitting atom and the environment. The plasma temperature can reach to thousands Kelvin. The major advantages of LIBS are: no sample preparation is required, fast, real-time measurements, depth profiling and spatial resolution and relatively simple set up compared to other conventional techniques [4] [5] [6]. LIBS has been widely used as a powerful technique for detection and monitoring of traces of elements of matter in all states of solid, liquid and gas in variety of applications such as industrial for metal detection in water [7] [8], analysis of minerals [9], environmental for identification of bacteria [10] [11], and biomedical such as analysis of cancerous tissues [12] [13] and dental caries [14] [15]. 
Interestingly, iron oxides are present in living organism where $\mathrm{Fe}$ (II) with an electron configuration of $1 s^{2} 2 s^{2} 2 p^{6} 3 s^{2} 3 p^{6} 3 d^{6} 4 s$ is responsible for transport of oxygen and storage of oxygen by means of haemoglobin and myoglobin. Magnetite $\left(\mathrm{Fe}_{3} \mathrm{O}_{4}\right)$ phase containing both $\mathrm{Fe}$ (II) and $\mathrm{Fe}$ (III) with electron configuration of $1 s^{2} s^{2} 2 p^{6} 3 s^{2} 3 d^{5}$ has iron ions arranged in octahedral (Oh) and tetrahedral (Th) sites which form the face-cent red cubic unit cell in the presence of oxygen. The structure of this phase can be represented as $[\mathrm{Fe}]_{\mathrm{Th}}\left[\mathrm{Fe}^{3+} \mathrm{Fe}^{2+}\right]{ }_{\mathrm{Oh}} \mathrm{O}_{4}$. In the inverse spinel structure of magnetite, $\frac{1}{4}$ of the octahedral holes are occupied by $\mathrm{Fe}^{2+}$ ions while the $\mathrm{Fe}^{3+}$ ions are equally divided between $\frac{1}{8}$ of the tetrahedral holes and $\frac{1}{4}$ of the octahedral holes; electron spins of $\mathrm{Fe}^{3+}$ ions in octahedral holes are aligned anti-parallel to those in tetrahedral holes while the $\mathrm{Fe}^{2+}$ ions tend to align their spins parallel with those of $\mathrm{Fe}^{3+}$ ions in adjacent octahedral sites, leading to a net magnetization. When the size of these nanoparticles becomes so small that their dimension can be considered as a single domain, they lack a hysteresis loop and a possession high field irreversibility, high saturation field and extra anisotropy contributions, it is called superparamagnetic nanoparticles (SPION) [16] [17]. Since, SPIONs posses remarkable size and morphology-dependent physical and chemical properties and obey the Coulomb's law and are easily controlled by an external magnetic field, thus they can be utilized for various biomedical applications such as hyperthermia [18] [19] drug delivery [20] [21] bioimaging [22] [23] and as nanofluid for heat transfer [24] [25].

However, synthesis and characterization of high-quality magnetic iron oxide nanoparticles (MNPs) has always been a major issue particularly when dealing with ultrafine particles to control their size at the nanometric scale due to the high surface energy. In this view, the elemental analysis of NPs could be very beneficial particularly at the stage of synthesis of NPs such as the ratio of elements or where the information about physicochemical forms of an element are highly regarded because the redox state of an element in solution can be toxic, adsorption behavior, and transport mechanism. Information about various oxidation states of the elements in variety of objects is crucial and challenging in analytical chemistry as for instance, natural waters contain iron in oxidation states +2 and +3 and Fe III content is significantly higher than Fe II content and it strongly affects the solubility of iron in water [26] [27]. Also, the iron oxide present in soil could form from an Fe II chloride solution in ambient temperatures and close to neutral $\mathrm{pH}$ values [28] and since oxidation leads to the formation of minerals such as goethite, an iron oxyhydroxide containing ferric iron, knowledge of Fe II: Fe III ration is important in this case. The commonly applied analytical methods are normally time consuming and require reagents and often produce inaccurate results in the presence of high Fe III content. Therefore, using LIBS as a non-contact, fast processing, and accurate technique would be de- 
sirable. Some researchers have employed LIBS to analyze the oxidation state of wine to monitor its quality [29], to measure the concentration of magnetite $\left(\mathrm{Fe}_{3} \mathrm{O}_{4}\right)$ nanoparticles (MNPs) in powder form in biocompatible matrix using $\mathrm{Nd}$ :YAG laser [30], or to analyze the graphene nanoparticles using $800 \mathrm{~nm}$ laser [31]. To best of our knowledge, no results are reported in the literature regarding the application of LIBS to magnetite nanoferrofluid synthesized at elevated temperature using Nd:YAG laser second harmonic generation wavelength $(532 \mathrm{~nm})$. Following our recent reports on MNPs [25] [32] [33] [34] [35], our goal in this work is to use pulse $532 \mathrm{~nm}$ laser to perform LIBS: 1) to investigate the emission lines of synthesized magnetite nanoferrofluid, 2) to determine the electron density and the temperature of dominant species, 3) to compare the elements ratio obtained at room and elevated temperatures, and 4) to study the effect of laser pulse frequency on elements ratio at different temperatures.

\section{Materials and Methods}

\subsection{Synthesis of Nanoferrofluid}

Solutions of $0.012 \mathrm{M}$ ferric chloride hexahydrate $\left(\mathrm{FeCl}_{3} \cdot 6 \mathrm{H}_{2} \mathrm{O}, 99 \%\right.$, Merck) and $0.006 \mathrm{M}$ ferrous sulfate heptahydrate $\left(\mathrm{FeSO}_{4} \cdot 7 \mathrm{H}_{2} \mathrm{O}, 99 \%\right.$, Merck) were prepared as iron source in double distilled water. The mixture of ferric and ferrous solution was then deoxygenated by bubbling $\mathrm{N}_{2}$ gas and sonicated for 30 minutes to prevent it from oxygenation. An amount of $0.9 \mathrm{M}$ concentration of ammonia solution was used as alkaline source and vigorously stirred under $\mathrm{N}_{2}$ bubbling at room temperature. The mixture of ferric and ferrous solution was drop wisely added to ammonia solution and magnetically stirred at $1500 \mathrm{rpm}$ for $30 \mathrm{~min}$ at ambient temperature. The colour of reaction mixture was immediately changed to back which is characteristic of a typical black nanoferrofluid material. The molar ratio of $\mathrm{Fe}^{2+}: \mathrm{Fe}^{3+}=1: 2$ and complete precipitation of $\mathrm{Fe}_{3} \mathrm{O}_{4}$ between $7.5-14 \mathrm{pH}$ was maintained under a non-oxidizing environment since this would critically affect the physical and chemical properties of the nanosized magnetic particles. The reaction temperature was kept constant at $25^{\circ} \mathrm{C}$ for 1 hour then the black precipitated mixture was purified using magnetic separation 5 times and sedimented by centrifugation. The resultant material was dried by freeze dryer for 24 hours.

\subsection{Characterization}

The crystalline phase of SPIONs was determined using $\mathrm{x}$ ray diffraction using $\mathrm{Cu}$ K $\alpha$ radiation (XRD, $\lambda=1.5406 \AA$, FK60-40 X-ray diffractometer). Particle size and morphology of these nanoparticles were determined by transmission electron microscopy (TEM, Philips CM-200-FEG microscope, $120 \mathrm{KV}$ ). The saturation magnetization of samples was measured by vibrating sample magnetometer (VSM-PAR 155) at $300 \mathrm{~K}$ under magnetic field up to $8 \mathrm{kOe}$. The plasma was analyzed using a spectrometer (UV-Vis USB 2000, Ocean Optics) equipped with 2048-pixel linear silicon CCD array $(14 \mu \mathrm{m} \times 200 \mu \mathrm{m})$ with a detector range of $200-1100 \mathrm{~nm}$, optical resolution of $0.1-10 \mathrm{~nm}$ FWHM connected to com- 
puter.

\section{Results and Discussion}

Figure 1(a) shows the TEM of synthesized SPIONs where polydispersity and some degree of aggregation, which mainly depends on the surface to volume ratio and augmentation of magnetic dipole-dipole interactions can be seen [32]. It is known that at room temperature,300 K, the critical size which defines the superparamagnetic region is equal to $27 \mathrm{~nm}$ [36] which in our case as seen in Figure 1 (b), the NPs distribution covers a range between 4 - $22 \mathrm{~nm}$ with most dominant size at $12 \mathrm{~nm}$ which satisfies the above condition.

The chemical procedure for synthesis of $\mathrm{Fe}_{3} \mathrm{O}_{4}$ is as follow: The first step is the hydrolysis of iron salts as

$$
\begin{aligned}
\mathrm{FeSO}_{4}+2 \mathrm{H}_{2} \mathrm{O} & \rightarrow \mathrm{SO}_{4}^{2-}+2 \mathrm{H}^{+}+\mathrm{Fe}(\mathrm{OH})_{2} \\
2 \mathrm{FeCl}_{3}+6 \mathrm{H}_{2} \mathrm{O} & \rightarrow 6 \mathrm{Cl}^{-}+6 \mathrm{H}^{+}+2 \mathrm{Fe}(\mathrm{OH})_{3} \\
\mathrm{Fe}(\mathrm{OH})_{3} & \rightarrow \alpha-\mathrm{FeOOH}+\mathrm{H}_{2} \mathrm{O}
\end{aligned}
$$

In a reactive system, $\mathrm{NH}_{4} \mathrm{OH}$ can be written as

$$
\mathrm{NH}_{4} \mathrm{OH} \rightarrow \mathrm{NH}_{4}^{+}+\mathrm{OH}^{-}
$$

The interaction between the resultant materials in solid state will lead to the
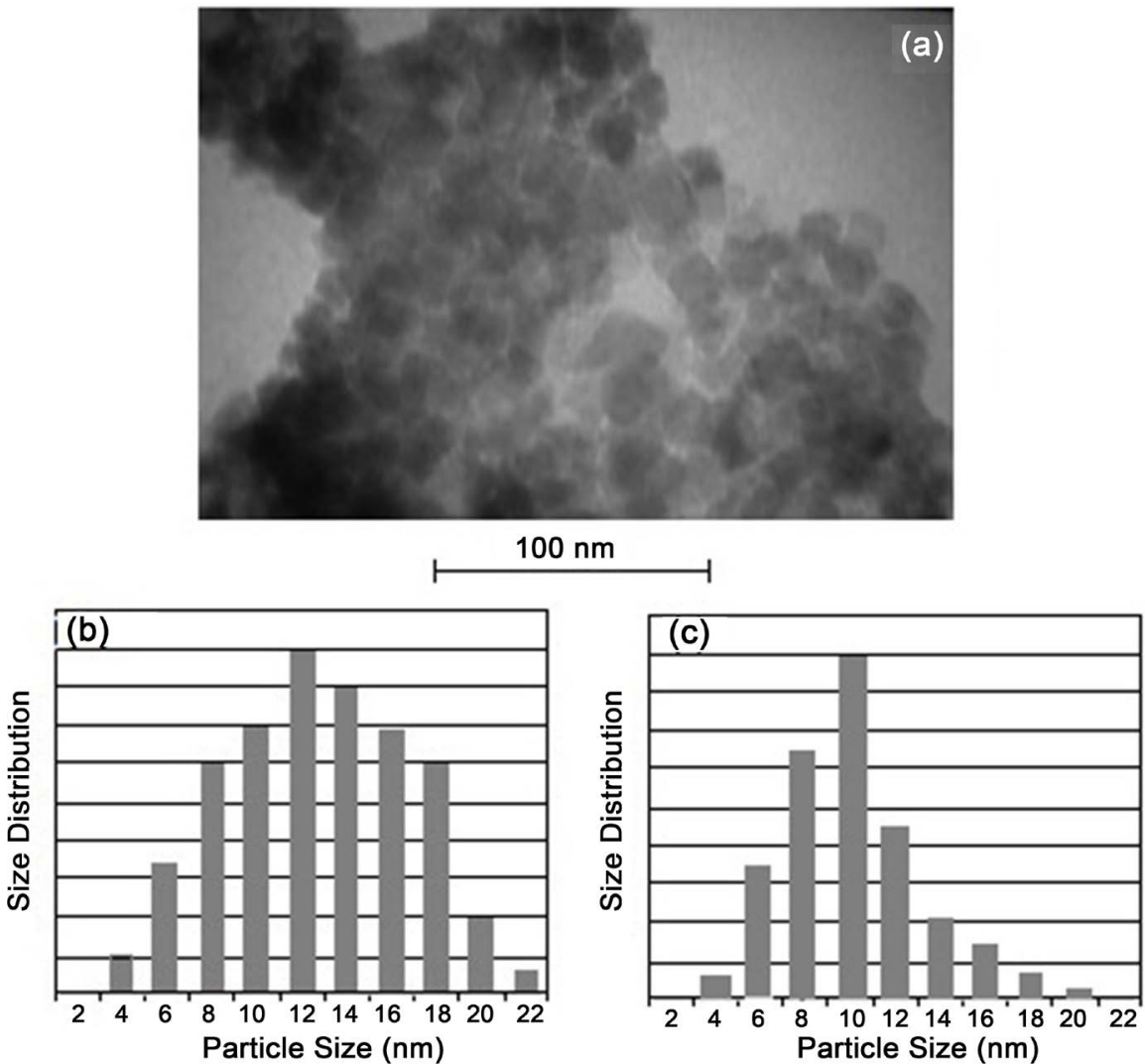

Figure 1. (a) An example of TEM micrograph of MNPs; (b) Size distribution of synthesized. MNPs at room temperature $\left(25^{\circ} \mathrm{C}\right)$; and (c) At elevated temperature $\left(70^{\circ} \mathrm{C}\right)$. 
formation of magnetite nanoparticles.

$$
2 \mathrm{FeOOH}+\mathrm{Fe}(\mathrm{OH})_{2}+8 \mathrm{OH}^{-}+8 \mathrm{H}^{+} \rightarrow \mathrm{FeO}_{4}+10 \mathrm{H}_{2} \mathrm{O}
$$

and the interaction of $\mathrm{NH}_{4}^{+}$cations with the resultant anions from hydrolysis of iron salts gives

$$
8 \mathrm{NH}_{4}^{+}+6 \mathrm{Cl}^{-}+\mathrm{SO}_{4}^{2-} \rightarrow 6 \mathrm{NH}_{4} \mathrm{Cl}^{-}+\left(\mathrm{NH}_{4}\right) \mathrm{SO}_{4}
$$

By increasing the solution of iron salt to $\mathrm{NH}_{4} \mathrm{OH}$, the ions of $\mathrm{Fe}^{3+}$ will precipitate as ferrihydrite which is a hydrous ferric oxyhydroxide mineral and then react with $\mathrm{Fe}^{2+}$ to produce magnetite. It is noteworthy that Ostwald ripening effect (growth of larger particles at the expense of dissolving the smaller ones) and aggregation of NPs can affect the growth phenomena. The synthesis process that contains many intermediate products can influence the nucleation and growth phenomena. Once the alkaline species and iron salts are added together, some cations and anions such as $\mathrm{SO}_{4}^{2-}, \mathrm{Cl}^{-}, \mathrm{NH}_{4}^{+}$and other intermediate products including $\mathrm{Fe}(\mathrm{OH})_{2}, \mathrm{Fe}(\mathrm{OH})_{3}$ and $\alpha$-FeOOH are produced. The release and usage rate of these materials determine the rate of nucleation and growth of MNPs. Once the magnetite nuclei are produced, the ion-to-ion attachment, NPs aggregation or Ostwald ripening will cause the growth of NPs [36]. The XRD result of crystalline structure of MNPs is given in Figure 2(a) where it indicates the formation of highly purified magnetite phase of iron oxide with diffraction peaks at (111), (220), (311), (400), (422), (511), (440), (533), which are the
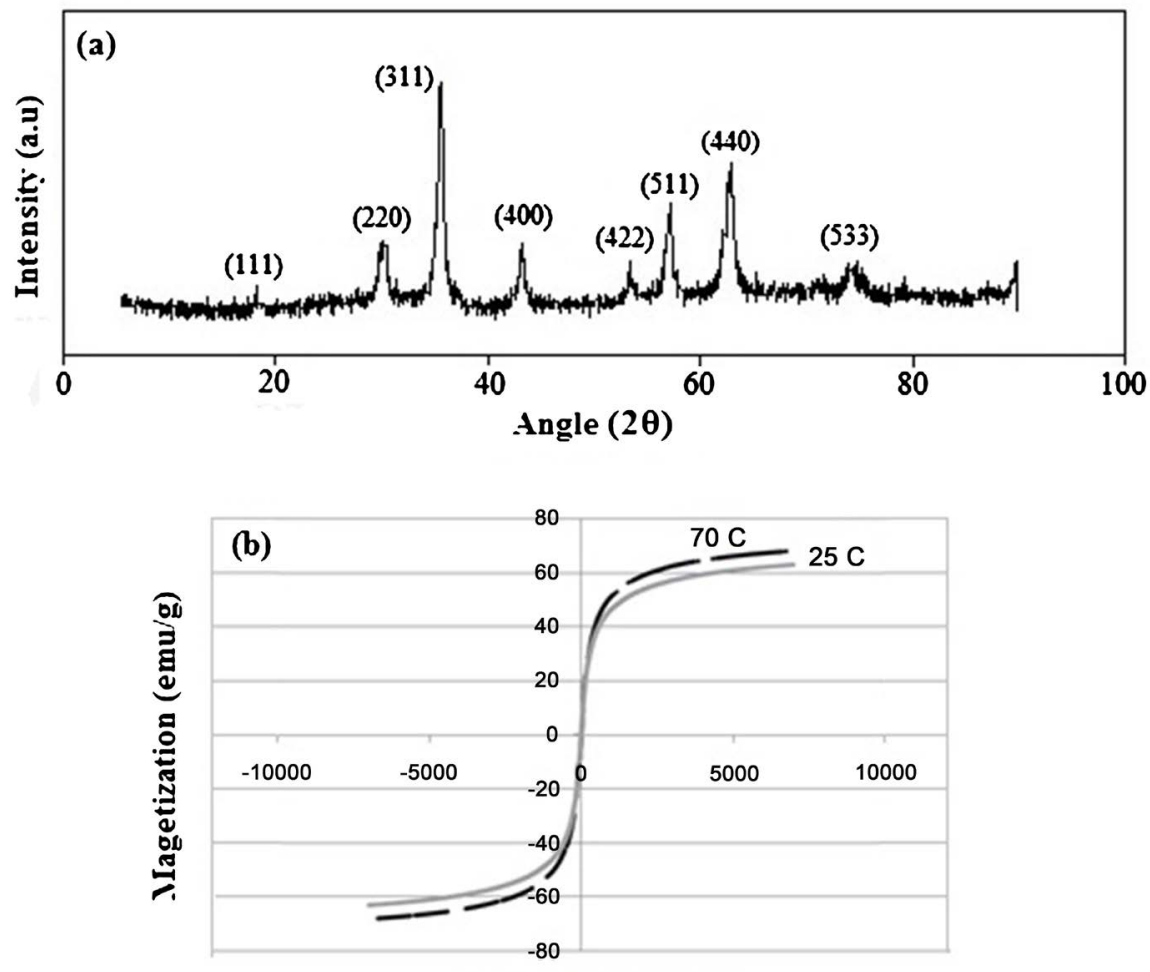

Applied Field (Oe)

Figure 2. (a) XRD pattern of MNPs; (b) VSM results of samples $\mathrm{S} 1$ and $\mathrm{S} 2$ at (a) $25^{\circ} \mathrm{C}$ and (b) $70^{\circ} \mathrm{C}$. 
characteristic peaks of magnetite nanoparticles $\left(\mathrm{Fe}_{3} \mathrm{O}_{4}\right)$ inverse spinel structure (JCPDS file No. 19-0629) without any interference with other phases of FexOy. Magnetic properties of MNPs were determined by VSM at room temperature. Figure 2(b) shows that the synthesized NPs exhibit strong magnetic properties of $63.1 \mathrm{emu} / \mathrm{g}$. However, these values can be lower than the bulk material of magnetite due to breaking of a large number of exchange bonds at the surface [32] [37]. Also, a complete reversibility of magnetization process confirms the superparamagnetic behaviour of prepared nanoparticles. Higher concentration of alkaline media increases the probability of non-magnetite layer production (i.e., magnetically dead layer) which in turn decreases the amount of saturation magnetization. Thus, there is a limit that alkaline media can have a positive effect on the saturation magnetization. Using Langevin's equation and magnetic experimental data, the average magnetic particle diameter was calculated [38]:

$$
a_{M}^{3}=\frac{18 K_{B} T}{\pi \mu_{0} M_{b} M_{s}}\left(\frac{\mathrm{d} M}{\mathrm{~d} H}\right)_{H \rightarrow 0}
$$

where $a_{M}$ is the magnetic particle diameter, $k_{B}$ is Boltzmann's constant, $\mu_{0}$ is the vacuum magnetic permeability, and $M_{b}$ is magnetization of bulk magnetite.

Figure 3 shows the experimental setup where the output of Q-switched Nd:YAG laser (LG-LM1) operating at second harmonic generation wavelength of $532 \mathrm{~nm}$ with a pulse duration of $8 \mathrm{~ns}$ was focused by a $100 \mathrm{~mm}$ lens into a 500 $\mu \mathrm{m}$ spot size on the surface of nanoferrofluid. The generated plasma was then detected by a $600 \mu \mathrm{m}$ core diameter optical fiber (LIBS-600-6-SR, Ocean Optics) connected to spectrometer (UV-Vis USB 2000, Ocean Optics) for analysis and the results were then displayed on computer. The pictures of LIBS at different laser energy were recorded by a fast CCD Camera (Panasonic Super Dynamic WV-CP450) connected to an optical microscope (Prior-UK). The examples of plasma recorded at different laser energy demonstrate that by increasing the input energy, the corresponding volume of plasma increases. Clearly, the emitted radiation in the visible spectral range originates from atomic and molecular

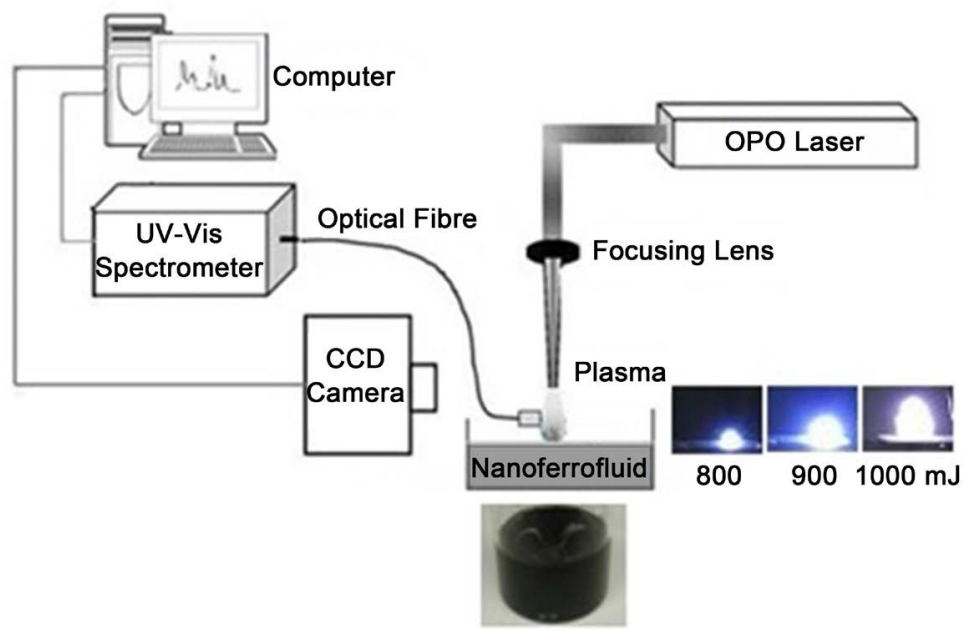

Figure 3. Experimental set up for LIBS. 
electronic transitions. Therefore, the heavy particles of low temperature plasma, the neutrals and their ions basically characterize the observed colour of a plasma. In our case, a very bright white spark indicating the high temperature plasma with blue shade probably because of ionized oxygen present in the surrounding environment was observed.

It is well-known that the laser wavelength and the pulse energy influence the interaction process where the former governs the amount of energy absorbed by the material and the plasma-material interaction and the latter, mainly controls the amount of ablated mas and the ablation rate. The plasma formation by nanosecond pulses as in our case seen in Figure 3, is determined by two major mechanisms of inverse Bremsstrahlung where the free electrons absorb energy the laser beam during a collision and increases their kinetic energy. It is responsible for heating the plasma electrons and secondly, the photoionization where the excited species and the ground state atoms are excited with high energies. As seen in Figure 4(a), the main transitions at $860 \mathrm{~mJ}$ are Fe I, Fe II, Cl III, O II, Cl $\mathrm{I}, \mathrm{OV}$ and the amplitude of the peaks are relatively small and by increasing the pulse number not a significant difference was observed. A significant saturation has occurred at excitation wavelength and some lines are very close to other transitions such as OV at $654.37 \mathrm{~nm}$ to that of hydrogen alpha at $656.28 \mathrm{~nm}$, which might be due to hydrogen in nanoferrofluid. However, the line shown by spectrophotometer software is compared and denoted to the closest with minimum difference. At $1050 \mathrm{~mJ}$, the transition lines are the same but the peaks between $250-280 \mathrm{~nm}$ seem to be more differentiated as delineated by circle in Figure 4(b). This is mainly because at higher temperatures, the ablated material dissociates (i.e., breaks down) into excited ions and atomic species, the amplitude of lines is also slightly increased. At even higher pulse energy of $1260 \mathrm{~mJ}$,

(a)

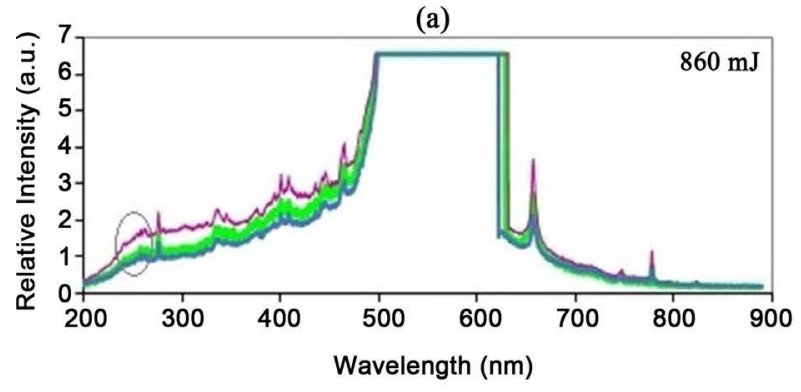

(b)

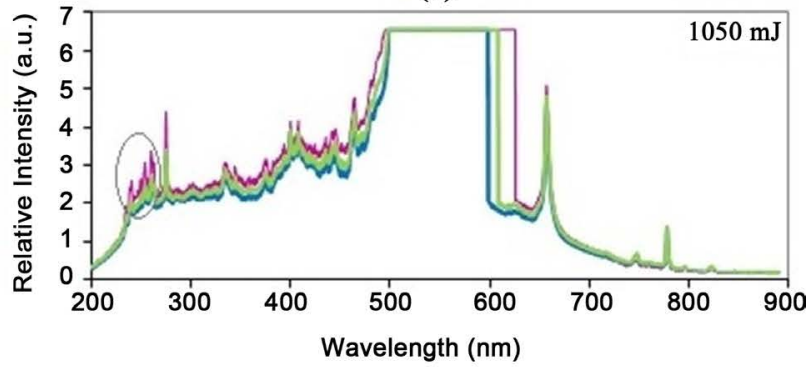

(c)

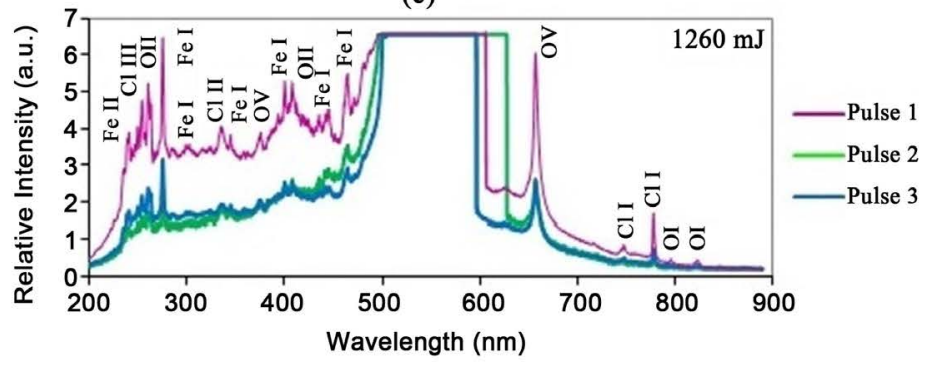

Figure 4. LIBS spectra at different laser pulse energy and pulse number. 
the first pulse caused much stronger lines amplitudes but then again are reduced at the consequent pulses, Figure 4(c). In principle, the loss of line intensity is mainly explained by the interaction of trailing portion of pulse with the plume and ejected particles which acts a shield hence a considerable fraction of energy is attenuated. The Fe III emission lines lie between $400-500 \mathrm{~nm}$, which are due to ${ }^{5} \mathrm{D}-{ }^{3} \mathrm{P},{ }^{5} \mathrm{D}-{ }^{3} \mathrm{~F},{ }^{5} \mathrm{D}-{ }^{3} \mathrm{G}$ and ${ }^{5} \mathrm{D}-{ }^{3} \mathrm{H}$ transitions among levels of the $3 \mathrm{~d}^{6}$ configuration (i.e., once it loses three electrons to form $3^{+}$, it becomes $1 s^{2} s^{2} 2 p^{6} 3 s^{2} 3 p^{6} 3 d^{5}$ ). These close emissions are mainly: He I $(400.92 \mathrm{~nm})$, Fe III $(400.83 \mathrm{~nm})$, O II $(466.16 \mathrm{~nm})$, Fe III $(465.80 \mathrm{~nm})$, Fe III $(527.04 \mathrm{~nm})$, and Fe II $(527.33 \mathrm{~nm})$. Thus, although there are some Fe III lines, only limited and most probable observable lines are indicated in the Figure 4 due to lack of sufficient space. The details of spectra analysis are given in Table 1 [39].

The central wavelength of line emission $\lambda_{0}$ is defined by the photon energy difference $\Delta E=E_{2}-E_{1}$ where $E_{1}$ and $E_{2}$ correspond to the energy of photons at longer and shorter detected wavelengths respectively (i.e., lower and upper energy levels):

$$
\lambda_{0}=h c / E_{2}-E_{1}
$$

where $h \approx\left(6.63 \times 10^{-34} \mathrm{Js}\right)$ is Planck constant Js and $c=3 \times 10^{8} \mathrm{~ms}^{-1}$ is the speed

Table 1. Spectral data of identified elements during LIBS.

\begin{tabular}{ccccc}
\hline \multirow{2}{*}{ Element } & $\begin{array}{c}\text { Wavelength } \\
( \pm 2 \mathbf{n m})\end{array}$ & $\begin{array}{c}\text { Transition Probability } \\
\mathbf{A}\left(\mathbf{s}^{-1}\right) \mathbf{1 0}\end{array}$ & \multicolumn{2}{c}{ Statistical Weight } \\
\cline { 4 - 5 } Fe II & 240.06 & $5.2 \mathrm{E}+00$ & $\mathbf{g}_{\mathrm{i}}$ & $\mathbf{g}_{\mathrm{k}}$ \\
Cl III & 253.25 & $5.3 \mathrm{E}+00$ & 12 & 14 \\
O II & 257.14 & $1.15 \mathrm{E}-01$ & 2 & 6 \\
Fe I & 273.35 & $8.6 \mathrm{E}-01$ & 11 & 9 \\
Fe I & 297.32 & $1.8 \mathrm{E}-01$ & 7 & 9 \\
Cl II & 332.91 & $1.5 \mathrm{E}-00$ & 5 & 7 \\
Fe I & 342.42 & $2 \mathrm{E}-01$ & 7 & 7 \\
OV & 376.15 & $1.6 \mathrm{E}-02$ & 7 & 5 \\
Fe I & 399.80 & $2.6 \mathrm{E}-02$ & 11 & 9 \\
O II & 408.46 & $7.2 \mathrm{E}-02$ & 6 & 8 \\
Fe I & 435.27 & $3.9 \mathrm{E}-02$ & 3 & 5 \\
Fe I & 444.23 & $3.7 \mathrm{E}-02$ & 5 & 5 \\
Fe I & 461.92 & $4.7 \mathrm{E}-02$ & 7 & 5 \\
OV & 654.37 & $1.64 \mathrm{E}-02$ & 5 & 5 \\
Cl I & 754.71 & $1.2 \mathrm{E}-01$ & 4 & 4 \\
Cl I & 776.92 & $6 \mathrm{E}-2$ & 6 & 6 \\
O I & 799.50 & $5.6 \mathrm{E}-04$ & 5 & 7 \\
O I & 822.18 & $2.89 \mathrm{E}-01$ & 7 & 7 \\
\hline & & & & 5 \\
\hline
\end{tabular}


of light. Since, the energy of a transition is characteristic of an element thus, the central wavelength represents the radiating species. The line intensity is quantified by the line emission coefficient in the unit of $\mathrm{W}\left(\mathrm{m}^{2} \mathrm{sr}\right)^{-1}$.

$$
\varepsilon_{21}=n(p) A_{21} \frac{h c}{4 \pi \lambda_{0}}=\int_{\text {line }} \varepsilon_{\lambda} \mathrm{d} \lambda
$$

where $n(p)$ is the species population or density in the excited state, $\mathrm{A}_{21}$ is the transition probability and $4 \pi$ represents the solid angle $\mathrm{d} \Omega$ i.e., isotropic radiation, measured in sr. Since, the line profile $p_{\lambda}$ correlates the emission coefficient with the spectral line emission coefficient we have:

$$
\varepsilon_{\lambda}=\varepsilon_{21} P_{\lambda} \text { with } \int_{\text {line }} P_{\lambda} \mathrm{d} \lambda
$$

The full width half maximum (FWHM) of the intensity is a characteristic of the line profile, which in turn depends on the broadening mechanism such as Doppler broadening where the profile has a Gaussian shape.

Since, the evaluation of plasma temperature is difficult due to its short lifetime and partly because of high temperature but one can use the Saha-Boltzmann equation for two or more consecutive spectral lines of the same species where the maximum and minimum amplitudes for each element can be used to measure the corresponding temperature [5]

$$
\frac{I_{1}}{I_{2}}=\frac{A_{1} g_{1} \lambda_{2}}{A_{2} g_{2} \lambda_{1}} \exp \left(-\left[\frac{E_{2}-E_{1}}{K T}\right]\right)
$$

Using the Equation (8) to determine $\Delta E$ and the values given in Table 1 are substituted in Equation (11) to determine the temperature of the species. Using the spectral data at $1050 \mathrm{~mJ}$ as the medium energy value, the plasma temperature was determined between $3881 \pm 200 \mathrm{~K}$ (or $0.33 \mathrm{eV}$ ) for Fe I and the maximum of $26,047 \pm 200 \mathrm{~K}$ (or $2.24 \mathrm{eV}$ ) for OV elements. Assuming that all the laser pulse energy is expended for ionization of MNPs, the number of free electrons corresponding to the element can be approximately calculated as $\mathrm{Ne}^{-} \approx(\mathrm{Ep} / \mathrm{Pi}) / \mathrm{eV}$ where Ep and $\mathrm{Pi}$ are laser pulse energy and the element ionization potential respectively [40]. The results in Figure 5 show a range of electron density spanning between $(0.47-6.80) \times 10^{17},(0.58-8.30) \times 10^{17}$ and $(0.69-9.96) \times 10^{17}$ at 860,1050 and $1260 \mathrm{~mJ}$ respectively where in each range OV and Fe I correspond to highest and lowest values. However, a rough estimate of the plasma volume $\approx$ 1000,1500 and $2000 \mu \mathrm{m}^{3}$ can be obtained from magnification of pictures taken by CCD camera in Figure 1, which effectively changes the electrons number to an order of $10^{20} \mathrm{~cm}^{-3}$ in each case.

Generally, the angular distribution of the ablated material from the target can be represented by the equation $I(\theta)=I_{0} \cos n \theta$, where $I(\theta)$ is the flux intensity along a direction forming an angle $\theta$ with the normal to the target surface. $I_{0}$ is initial intensity corresponding to $\theta=0$ and $n$ is parameter related to the anisotropy of the distribution. When $n=1$, it corresponds to a perfectly spherical distribution and $n>1$ implies a more directional plume shape as in our case in 


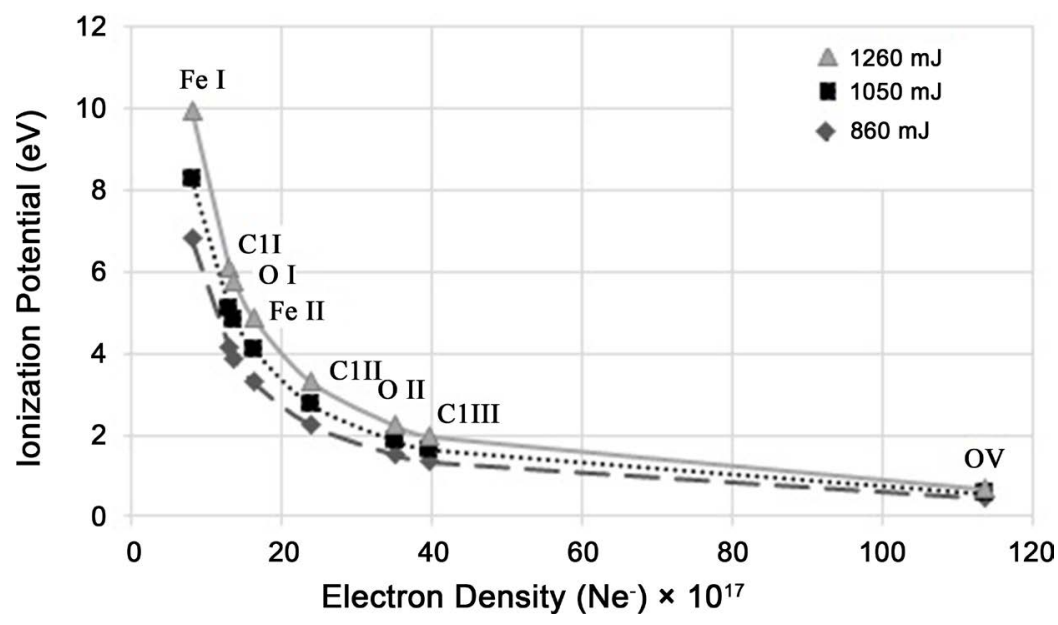

Figure 5. Calculated electron density of elements as a function of ionization potential.

Figure 1 particularly at higher energy levels. Indeed, the plasma plume is a combination of hot electrons, colder heavier ions and neutral atoms, which results in an inhomogeneous temperature distribution. The interaction of hot plasma with cold region at the interface with the surrounding colder air may destroy the shape of the emitted lines which can appear as a decrease of the spectral line intensity and increase of the width of line at FWHM [40] [41]. Also, upon rapid expansion of the plasma both its temperature and recoil pressure decrease which reduce the rate of heat transfer to the surface. Also, according to Serov and Richardson [42] an intense magnetic field due to laser-induced plasma displays a $1 / r^{2}$ radial dependence and that such fields $\left(10^{9} \mathrm{Wcm}^{-2}\right)$ would significantly affect the electron thermal conduction process. Thus, it has a crucial role in determining the temperature and density distribution within the plasma. It is also interesting to note that the plasma temperature and hence its possible consequent effects can influence the quality of the material surface during the interaction process. For example, the high pressure exerted on the surface by the plasma plume can suppress the vaporization of the material by raising the boiling point of the material well beyond its normal value and as the plasma expands it generates an impulse reaction on the surface thus, radiates part of its thermal energy back to the surface. Consequently, a high temperature is maintained for a short time after the laser pulse has ended. It is expected that the high temperature is transferred to the surface by high velocity electrons via both direct conductivity and radiative transfer [25] [43].

Figure 6 demonstrates the variation of elements ratio with energy for different temperatures at constant frequency of $1 \mathrm{~Hz}$ where the higher pulse numbers produced a ratio with higher signal intensity. A characteristic behaviour of the results is their repeating systematic zig-zag feature at almost the same values of energy. The ratio of elements for samples synthesized at room temperature (S1) is higher than those at elevated temperature (S2) except in the case of Fe III/OII where the results are almost superimposed.

At higher frequency $(3 \mathrm{~Hz})$ shown in Figure 7 , the ratio of elements remained 

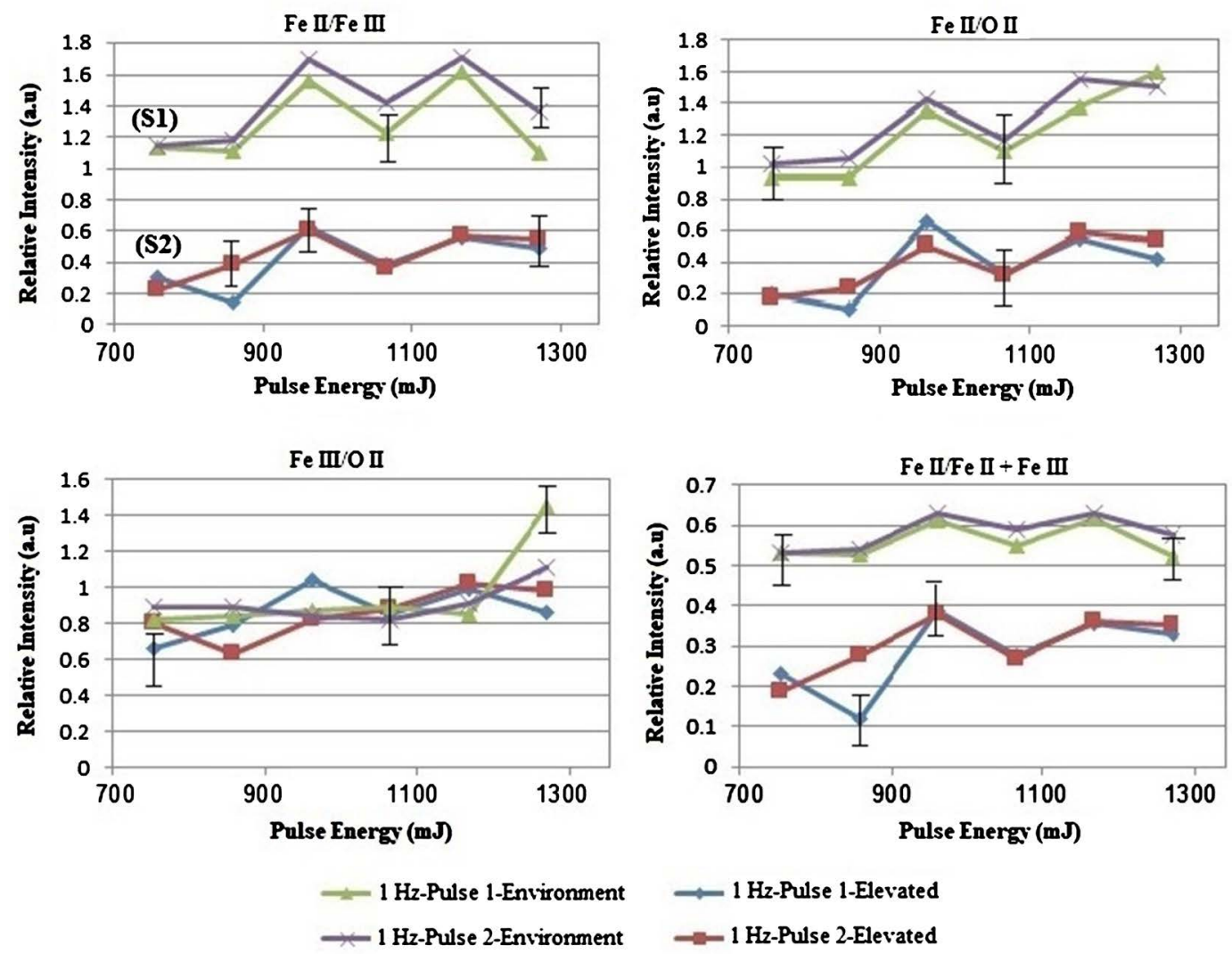

Figure 6. Variation of elements ratio for S1 and S2 with pulse energy at $1 \mathrm{~Hz}$.
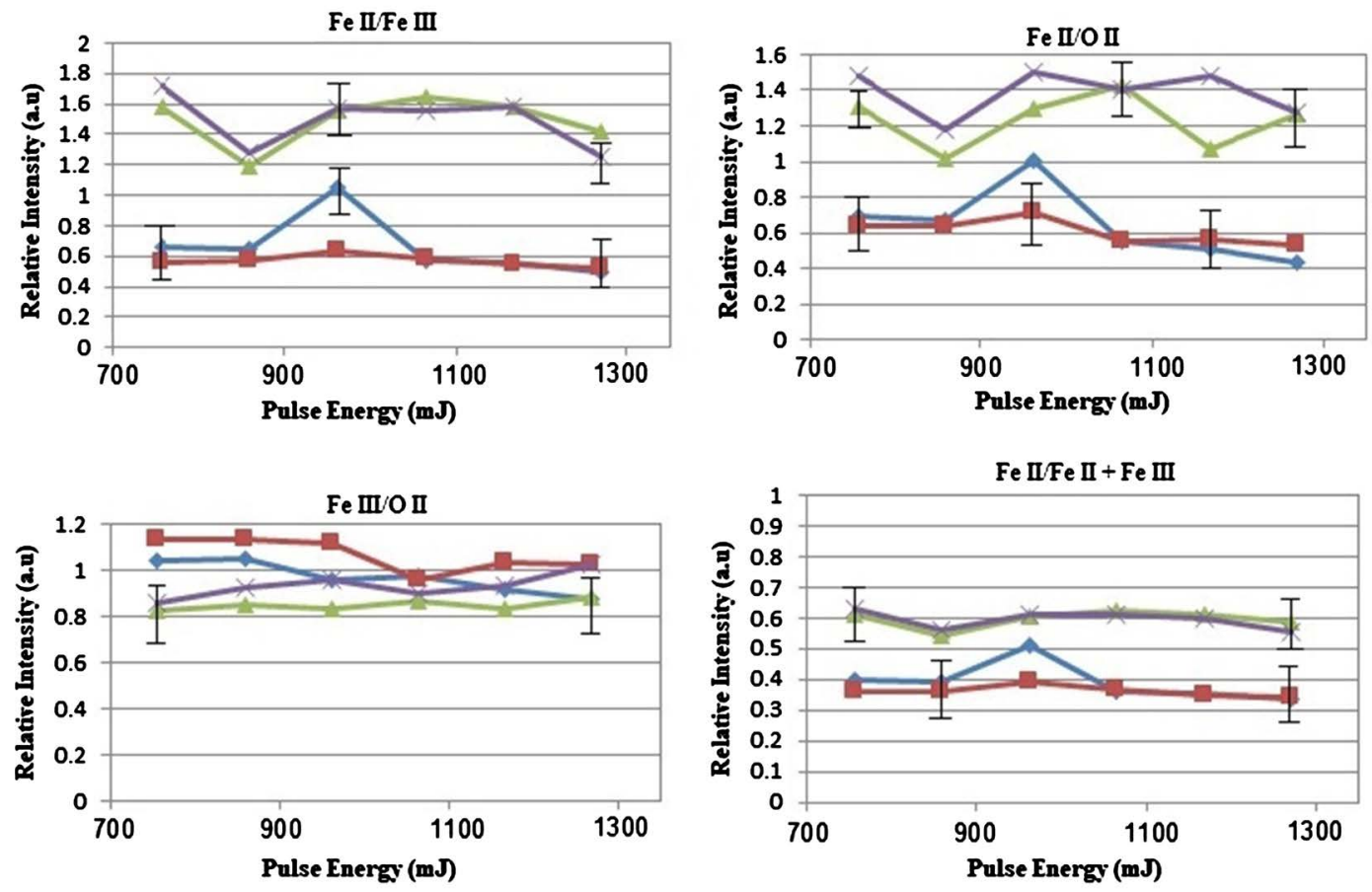

$-3 \mathrm{~Hz}$ (3 pulses)-Environment

$\longrightarrow 3 \mathrm{~Hz}$ (3 pulses)-Elevated

$\longrightarrow 3 \mathrm{~Hz}$ (6 pulses)-Environment

$-3 \mathrm{~Hz}$ (6 pulses)-Elevated

Figure 7. Variation of elements ratio for S1 and S2 with pulse energy at $3 \mathrm{~Hz}$. 
almost at the same values for S1 samples but it was increased from an average value of 0.4 to 0.6 for Fe II/Fe III and Fe II/ Fe/OII. Again, the similar behaviour was obtained in the case of Fe III/OII i.e., no significant difference. Interesting enough, the ratio for Fe II/Fe II + Fe III was reduced from 1.4 to 0.6 which is very close to desirable value. A similar to results to $(3 \mathrm{~Hz})$ was obtained at $6 \mathrm{~Hz}$ for both S1 and S2 at $6 \mathrm{~Hz}$ shown in Figure 8.

The highlights of the above observations are summarized as plots shown in Figure 9 where it demonstrates that at constant pulse energy (i.e., $860 \mathrm{~mJ}$ ), the ratio increases with frequency for both samples of S1 and S2 with 1.1 lowest at 1 $\mathrm{Hz}$ and highest about 1.3 at $3 \mathrm{~Hz}$ for S1 (Figure 9(a1)) and 0.2 at $1 \mathrm{~Hz}$ and 0.6 at $3 \mathrm{~Hz}$ for S2 (Figure 9(a2)) respectively. As in the case of Fe II/Fe II + Fe III, significantly lower values were achieved for both S1 and S2 i.e., about 0.55 for S1 (Figure 9(b1)), and 0.1 at $1 \mathrm{~Hz}$ for S1 (Figure 9(b2)) and about 0.3 at 3 and 6 $\mathrm{Hz}$ respectively (Figure 9(b2)). Despite the similar behaviour at higher energy $(1260 \mathrm{~mJ})$, a noticeable change was observed at $6 \mathrm{~Hz}$ both for S1 and S2 (Figure 9(c2) and Figure 9(d2)) where the ratio showed an increasing trend up 1 and 0.5 for S1 and S2 respectively whereas in the case of $860 \mathrm{~mJ}$ it reached the plateau at $6 \mathrm{~Hz}$ indicating that at higher pulse energy more transition lines corresponding to Fe II is produced. However, no firm comment can be made about its linear extrapolation as the experiment was not performed beyond this value. It is suggested for the future research to consider the effects of temperature and plasma formation on the quality of nanoferrofluid composition to ensure its
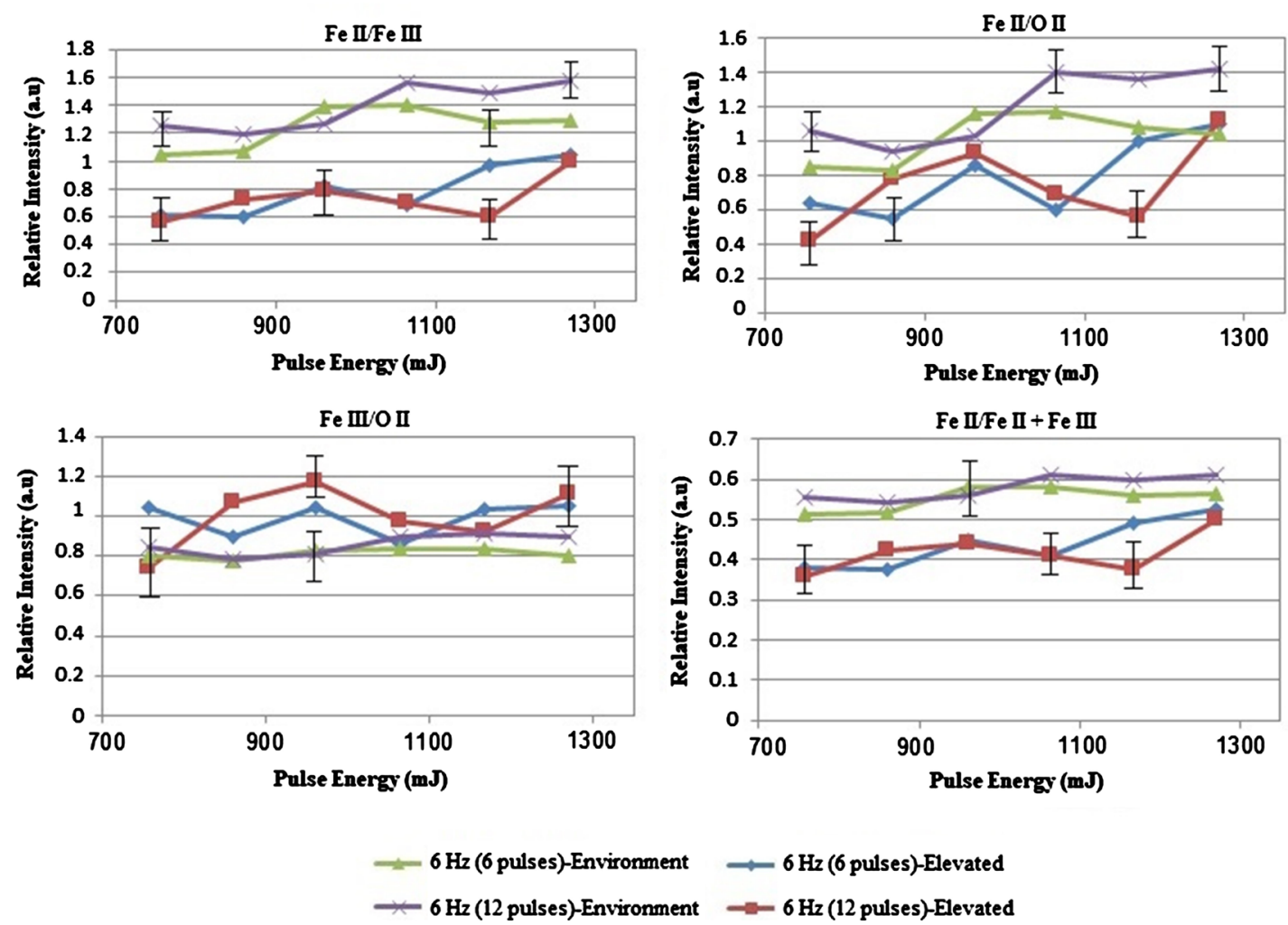

Figure 8. Variation of elements ratio for S1 and S2 with pulse energy at $6 \mathrm{~Hz}$. 


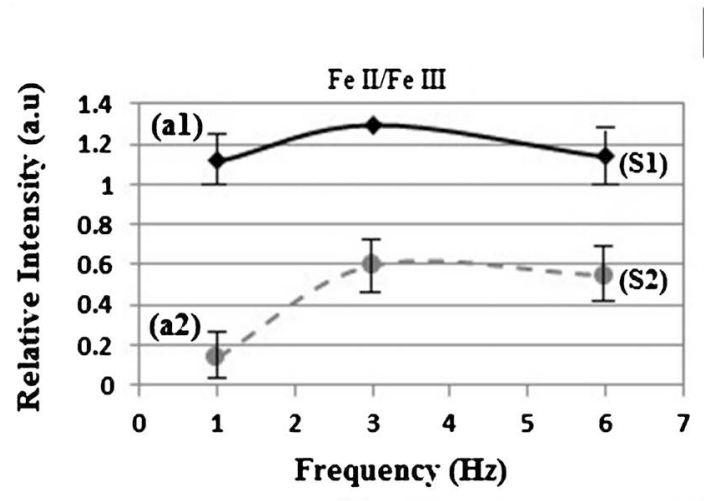

\section{$860 \mathrm{~mJ}$}
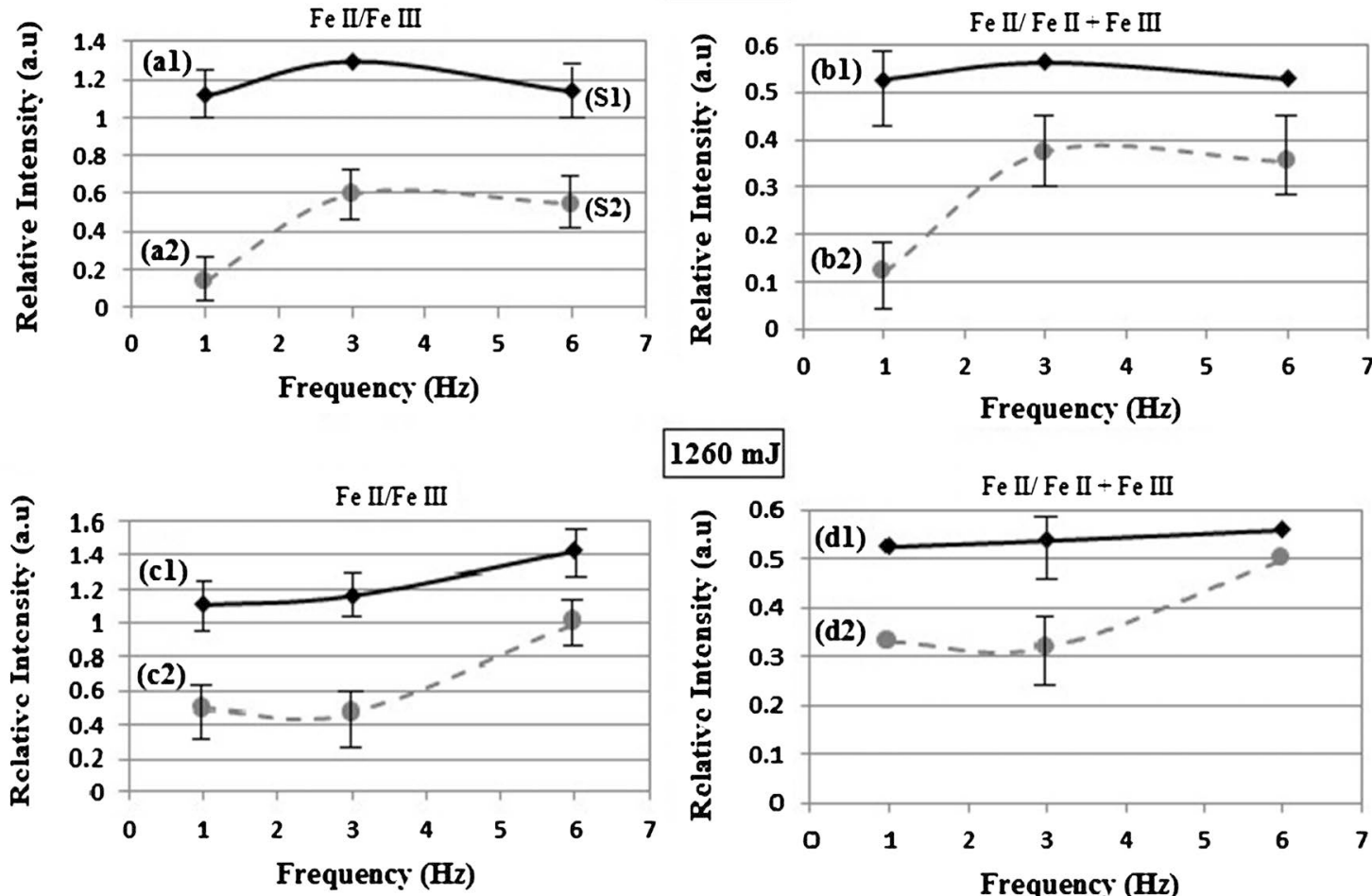

\section{$1260 \mathrm{~mJ}$}

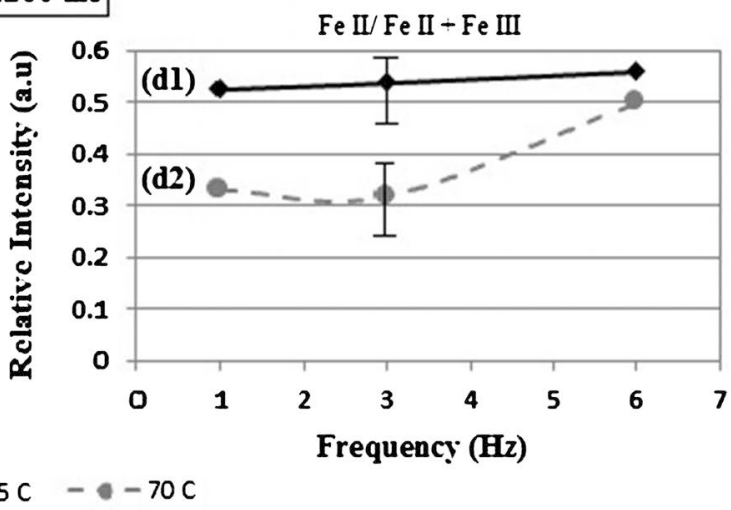

Figure 9. Variation of Fe II:Fe III and Fe II:Fe II + Fe III for S1 and S2 with frequency at different energy.

structural integrity with respect to its applications to avoid any possible damage.

\section{Conclusion}

Elemental analysis of magnetite $\left(\mathrm{Fe}_{3} \mathrm{O}_{4}\right)$ nanoferrofluid was investigated at two different temperatures using $532 \mathrm{~nm}$-based LIBS technique. Using Saha-Boltzmann relation, the transition lines temperature and the corresponding electron density were calculated. It was shown that the constituting elements and their ratio can be identified depending on the synthesizing and optical conditions. The most important outcome of the experiment is that the elemental ratio is significantly lower at elevated temperature than those at room temperature at all laser energy. Equally, at higher frequency and room temperature no considerable change in the ratio was observed, however, at elevated temperature and $860 \mathrm{~mJ}$, the ratio was increased and reached its maximum value at $3 \mathrm{~Hz}$. This was not observed at $1260 \mathrm{~mJ}$. One practical application where such an approach can be beneficial and plays a key role in number of scientific, engineering and biomedical applications is when one requires to determine and adjust the ratio of Fe II:Fe III or Fe II/Fe II + Fe III during the synthesis process.

\section{Acknowledgements}

The authors are thankful to AUT for the support and Faculty Funding for this research work. 


\section{Conflicts of Interest}

The authors declare no conflicts of interest regarding the publication of this paper.

\section{References}

[1] Durrant, S.F. and Ward, N. (2005) Recent Biological and Environmental Applications of Laser Ablation Inductively Coupled Plasma Mass Spectrometry (LA-ICP-MS). Journal Analytical Atomic Spectrometry, 20, 821-829. https://doi.org/10.1039/b502206a

[2] Perez-Seeadilla, J.A., Jurdo-Lopeze, A. and Luque de Castero, M. (2007) Complementarity of XRFS and LIBS for Corrosion Studies. Talanta, 71, 97-102.

https://doi.org/10.1016/j.talanta.2006.03.034

[3] Benninghoven, A., Rudenauer, F.G. and Werner, H. (1987) Secondary Ion Mass Spectrometry-Basic Concepts, Instrumental Aspects, Applications and Trends. Wiley, New York.

[4] Griem, H.R. (1964) Plasma Spectroscopy. McGraw-Hill, New York.

[5] Lochet-Holtgreven, W. (1968) Plasma Diagnostics. North Holland Press, Amsterdam.

[6] Fantz, U. (2006) Basics of Plasma Spectroscopy. Plasma Sources Science Technology, 15, S137-S147. https://doi.org/10.1088/0963-0252/15/4/S01

[7] Ben Ahmad, J., Terzi, N. and Lakhdar, Z. (2002) Temporal Characterization of a Plasma Produced by Interaction of Laser Pulses with Water Solutions. Laser Chemistry, 20, 111-122. https://doi.org/10.1080/02786270215155

[8] Lee, Y., Oh, S.W. and Han, S.H. (2012) Laser-Induced Breakdown Spectroscopy of Heavy Metal Ions at the Sub-Parts per Million Level in Water. Applied Spectroscopy, 66, 1385-1396. https://doi.org/10.1366/12-06639R

[9] Sharma, Sh., Misra, A., Lucey, P. and Lentz, C. (2009) A Combined Remote Raman and LIBS Instrument for Characterizing Minerals with $532 \mathrm{~nm}$ Laser Excitation. Spectrochimica Acta Part A, 73, 468-476. https://doi.org/10.1016/j.saa.2008.08.005

[10] Rehse, S. (2009) Laser-Based Identification of Pathogenic Bacteria. The Physics Teacher, 47, 152-156. https://doi.org/10.1119/1.3081297

[11] Diedrich, J., Rehse, S. and Palchaudhuri, S. (2007) Pathogenic Escherichia coli Strain Discrimination Using Laser-Induced Breakdown Spectroscopy. Journal Applied Physics, 102, 014702. https://doi.org/10.1063/1.2752784

[12] Corsi, M., Cristoforetti, G., Hidalgo, M. and Legnaaioli, S. (2003) Applications of Laser-Induced Breakdown Spectroscopy Technique to Hair Tissue Mineral Analysis. Applied Optics, 42, 6133-6137. https://doi.org/10.1364/AO.42.006133

[13] Iman, H., Mohamed, R. and Eldakrouri, A. (2012) Primary Study of the Use of Laser-Induced Plasma Spectroscopy for the Diagnosis of Breast Cancer. Optics Photonics Journal, 2, 193-199. https://doi.org/10.4236/opj.2012.23029

[14] Nimez, S. (1994) Evaluation of Physical Parameters during the Plasma-Induced Ablation of Tooth. SPIE, 2323, 170-179.

[15] Khosroshahi, M.E. and Ghasemi, A. (2004) Interaction Studies of Multimode Pulsed HF Laser with Enamel Tissue using Photothermal Deflection and Spectroscopy. Laser Medical Science, 18, 196-203. https://doi.org/10.1007/s10103-003-0283-5

[16] Pankhurst, Q., Connolly, J., Jones, S. and Dobson, J. (2003) Applications of Magnetic Nanoparticles in Biomedicine. Journal Physics D. Applied Physics, 36, 167-181. 
https://doi.org/10.1088/0022-3727/36/13/201

[17] Gupta, A. and Gupta, M. (2005) Synthesis and Surface Engineering of Iron-Oxide Nanoparticles for Biomedical Applications. Biomaterials, 26, 3995-4021. https://doi.org/10.1016/j.biomaterials.2004.10.012

[18] Candido, N., Calmon, M., Taboga, S. and Bonilha, J. (2014) High Efficacy in Hyperthermia-Associated with Polyphosphate Magnetic Nanoparticles for Oral Cancer Treatment. Journal Nanomedicine Nanotechology, 5, 205.

[19] Khosroshahil, M.E., Ghazanfari, L., Hassannejad, Z. and Lenhert, S. (2015) In-Vitro Application of Doxorubicin Loaded Magnetoplasmonic Thermosensitive Liposomes for Laser Hyperthermia and Chemotherapy of Breast Cancer. Journal Nanomedicine Nanotechnology, 6, 298.

[20] Esterlich, J., Eseribano, E. and Queralt, J. (2015) Iron Oxide Nanoparticles for Magnetically-Guided and Magnetically-Responsive Drug Delivery. Molecular Science, 6, 8070-8080.

[21] Khosroshahi, M.E., Ghazanfari, L. and Hasannejad, Z. (2017) Effect of Laser Wavelengths on Drug Release with and Without Gold Nanoshells and Magnetic Guidance on Uptake by Cancer Cells. Journal Nanomedicine Research, 6, Article ID: 00152. https://doi.org/10.15406/jnmr.2017.06.00152

[22] McCarthy, J.R. and Weisseleder, R. (2008) Multifunctional Magnetic Nanoparticles for Targeted Imaging and Therapy. Advance Drug Deliver Review, 60, 1241-1251. https://doi.org/10.1016/j.addr.2008.03.014

[23] Khosroshahi, M.E. and Asemani, M. (2017) Synthesis, Characterization and Imaging of Fluorescein Isothiocyanate Conjugated Magnetite Nanoparticles in MCF 7 Breast Cancer Cell Lines. International Journal Nanomaterial Nanotechnology Nanomedicine, 3, 44-50.

[24] Shima, P.D. and Philip, J. (2014) Role of Thermal Conductivity of Dispersed Nanoparticles on Heat Transfer Properties of Nanofluid. Journal Industrial Engineering Chemistry Research, 53, 980-988. https://doi.org/10.1021/ie403086g

[25] Khosroshahi, M.E. and Asemani, M. (2017) Dynamics Study and Analysis of Laser-Induced Transport of Nanoferrofluid in Water Using Fluorescein Isothiocyanate (FITC) as Fluorescence Marker. Journal Modern Physics, 8, 2219-2244. https://doi.org/10.4236/jmp.2017.814137

[26] Didukh, S., Losev, V., Borodina, E. and Maksimov, N. (2017) Separation and Determination of Fe (III) and Fe (II) in Natural and Waste Waters Using Silica and Gel Sequentially Modified with Polyhexamethylene Guanidine and Tiron. Journal Analytical Methods in Chemistry, 2017, Article ID: 8208146. https://doi.org/10.1155/2017/8208146

[27] Rusevova, K., Kopinke, F. and Georgi, A. (2012) Nano-Sized Magnetic Iron Oxides as Catalysts for Heterogeneous Fenton-Like Reactions-Influence of Fe (II)/Fe (III) Ratio on Catalytic Performance. Journal Hazardous Materials, 241, 433-440. https://doi.org/10.1016/j.jhazmat.2012.09.068

[28] Taylor, R.M. (1980) Formation and Properties of Fe (II) Fe (III) Hydroxy-Carbonate and Its Possible Significance in Soil Formation. Clay Minerals, 15, 369-382. https://doi.org/10.1180/claymin.1980.015.4.04

[29] Danilewicz, H. (2016) Fe (II): FE (III) Ratio and Redox Status of White Wines. American Journal Enology Viticulture, 67, 1-3. https://doi.org/10.5344/ajev.2015.15088

[30] Brown, E. and Rehse, S. (2007) Laser-Induced Breakdown Spectroscopy of $\gamma-\mathrm{Fe}_{3} \mathrm{O}_{4}$ Nanoparticles in Biocompatible Alginate Matrix. Spectrochemica Acta B, 62, 1475-1483. 
https://doi.org/10.1016/j.sab.2007.10.023

[31] Ganeev, R.A. (2017) Enhancement of High-Order Harmonics Generated in Laser-Induced Plasma Using Ionic Resonances and Nanoparticles. Nonlinear Quantum Optics, 122, 250-268.

[32] Khosroshahi, M.E. and Ghazanfari, L. (2010) Preparation and Characterization of Silica-Coated Iron Oxide Bionanoparticles under $\mathrm{N}_{2}$ Gas. Physica E, 42, 1824-1829. https://doi.org/10.1016/j.physe.2010.01.042

[33] Khosroshahi, M.E., Nourbakhsh, M.S. and Ghazanfari, L. (2011) Synthesis and Biomedical Application of $\mathrm{SiO}_{2} / \mathrm{Au}$ Nanofluid Based on Laser-Induced Surface Plasmon Resonance Thermal Effect. Journal Modern Physics, 2, 944-953. https://doi.org/10.4236/jmp.2011.29112

[34] Khosroshahi, M.E. and Ghazanfari, L. (2012) Preparation and Rheological Studies of Uncoated and PVA-Coated Magnetite Nanofluid. Journal Magnetism and Magnetic Materials, 324, 4143-4146. https://doi.org/10.1016/j.jmmm.2012.07.025

[35] Tajabadi, M., Khosroshahia, M.E. and Bonakdar, Sh. (2013) An Efficient Method of SPION Synthesis Coated with Third Generation PAMAM Dendrimer, Colloids and Surface A. Physicochemical Engineering Aspects, 431, 18-26.

https://doi.org/10.1016/j.colsurfa.2013.04.003

[36] Xue, D., Chai, G., Li, X. and Fan, X. (2008) Effects of Grain Size Distribution on Coercivity and Permeability of Ferromagnets. Journal Magnetism and Magnetic Materials, 320, 1541-1543. https://doi.org/10.1016/j.jmmm.2008.01.004

[37] Lai, Z.T., Guang, Y.W., Jing, Q.X., Guo, Z.J. and Li, Y. (2007) Effects of Synthetical Conditions on Octahedral Magnetite Nanoparticles. Material Science Engineering $B, 136,101-105$.

[38] Racuciu, M. (2009) Synthesis Protocol Influence on Aqueous Magnetic Fluid Properties. Current Applied Physics, 9, 1062-1066.

https://doi.org/10.1016/j.cap.2008.12.003

[39] Lide, D.R. (1999) Hand Book of Chemistry and Physics. 80th Edition, CRC Press, London.

[40] Aguilera, J.A., Aragon, C. and Penalba, F. (1998) Plasma Shielding Effect in Laser Ablation of Metallic Samples and Its Influence on LIBS Analysis. Applied Surface Science, 127-129, 309-314.

[41] Angel, S.M., Stratis, D.N., Eland, K.L., Lai, T. and Berg, M.A. (2001) LIBS Using Dual- and Ultra-Short Laser Pulses. Fresenius Journal of Analytical Chemistry, 369, 320-327. https://doi.org/10.1007/s002160000656

[42] Serov, R. and Richardson, M. (1976) Measurement of Intense Magnetic Fields Associated with Laser-Produced Plasmas. Applied Physics Letter, 28, 115-118. https://doi.org/10.1063/1.88675

[43] Khosroshahi, M.E., Anoushepour, F., Hadavi, M. and Mahmoodi, M. (2010) In Situ Monitoring the Pulse $\mathrm{CO}_{2}$ Laser Interaction with 316-L Stainless Steel Using Acoustical Signals and Plasma Analysis. Applied Surface Science, 256, 7421-7427. https://doi.org/10.1016/j.apsusc.2010.05.083 